\title{
Performance Prediction of a Phase Synchronization Link for Bistatic SAR
}

\author{
Marwan Younis, Member, IEEE, Robert Metzig, and Gerhard Krieger, Member, IEEE
}

\begin{abstract}
Oscillator phase noise can dictate the performance of bi- and multistatic SAR imaging. In this paper the use of a dedicated synchronization link to quantify and compensate oscillator phase noise is investigated. Different synchronization schemes are presented and their performance is analyzed. The error contribution of the synchronization link itself, which may suffer from receiver noise, aliasing, interpolation and filter mismatch is included in the analysis. The synchronization link performance is given in a frequency domain closed integral form.
\end{abstract}

\section{INTRODUCTION}

In [1] it was shown that oscillator phase noise levels insignificant for monostatic SAR can severely degrade the performance of bistatic and multistatic SAR. Depending on the application, acceptable performance may be reached through highly stable oscillators, or by analyzing the processed SAR signal in conjunction with ground control points [1], [2]. Here, we investigate an alternative approach based on a hardware system to synchronize the two instruments. If a dedicated intersatellite synchronization link is established to exchange the oscillator signals, then, by adequate processing, a correction signal can be derived to compensate the oscillator phase noise in the bistatic SAR signal. The use of a continuous duplex intersatellite links for oscillator drift compensation was first proposed in [3] and has been further investigated in [4]. The concept is somehow similar to microwave ranging used to determine the separation between platforms [5], [6], however, the phase synchronization requires a different processing approach. In this paper we extend the approach to include additional synchronization schemes and we include additional factors such as receiver noise and Doppler effect as well as contributions known from sampling theory [7], such as aliasing and interpolation errors, which are crucial for the performance of the synchronization. Several contributions such as the influence the ionosphere or relativistic effects are neglected; we are aware that these contributions might become significant, however, we rather choose to maintain the general overview character of our approach.

The paper is organized as follows: after pointing out possible synchronization schemes and their respective timing issues in section II, the signal phase representation is stated and used to derive the compensation phase in section III. The performance of the synchronization link is analyzed in section IV with an increasing degree of complexity corresponding to the different synchronization schemes. The most general (complex) mathematical description will be the last covering the former cases.

Manuscript received ; revised

M. Younis, R. Metzig, and G. Krieger are with the Microwaves and Radar Institute, German Aerospace Centre (DLR). E-Mail: marwan.younis@dlr.de.

\section{SYNCHRONIZATION SCHEMES}

Depending on the SAR hardware and the affordable synchronization system complexity, various hardware configurations may be used to establish the synchronization link. In all cases the aim is to exchange signals containing information on the oscillator noise between the radar instruments.

The continuous duplex synchronization scheme which has first been suggested in [3] is shown in Fig. 1(a). In this case both satellite 1 and 2, separated by a distance $r$, continuously transmit and receive their local oscillator signals. Alternatively, pulsed synchronization is possible as indicated in Fig. 1(b). Here, each satellite repeatedly transmits its synchronization signal. For pulsed duplex synchronization, both satellites transmit synchronization pulses at the same time instances. In the case of pulsed alternate synchronization there is a time delay between the transmit instances of satellite 1 and 2 .

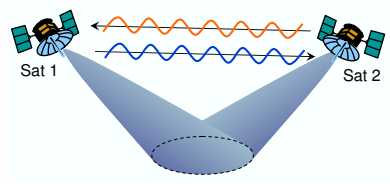

(a) continuous duplex

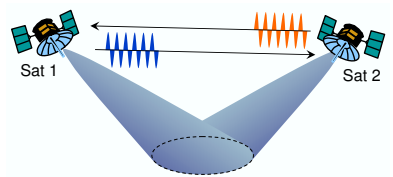

(b) pulsed (duplex/alternate)
Fig. 1. Synchronization schemes.

A parameterized timing diagram covering the above three cases is shown in Fig. 2. At time $t$ satellite 1 transmits the synchronization signal of duration $T_{\mathrm{p}}$, which is received $\tau_{12}$ seconds later by satellite 2. Similarly, after an internal system delay of $\tau_{\text {sys }}$, satellite 2 transmits its synchronization signal at $t+\tau_{\text {sys }}$, which is received by satellite 1 with a delay corresponding to the signal travel time $\tau_{21}$. This procedure may be repeated at the synchronization rate $f_{\text {syn }}$. The synchronization lasts over the data take time $T_{\text {data }}$.

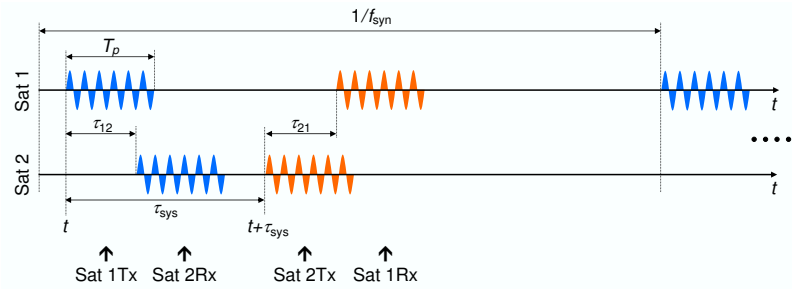

Fig. 2. Timing diagram for the exchange of synchronization pulses. 


\section{SYNCHRONIZATION SYSTEM MODEL}

The contribution of the oscillator phase noise to the SAR signal phase error can be compensated by establishing a method of phase referencing. Here, a direct exchange of synchronization signals between the satellites through a dedicated link is assumed. In order to keep the methodology independent of the specific synchronization scheme and link implementation a general mathematical model is established in this section. The aim is to use this model as a basis for deriving quantitative estimates for the performance of the synchronization link.

\section{A. Oscillator Signal Phase}

Satellite $i$ transmits a synchronization signal, which is received by satellite $j$, where $i, j \in\{1,2\}$. The frequency of oscillator $i$ at start of data take $t_{0}$ is $f_{i}=f_{0}+\Delta f_{i}$, with the nominal frequency $f_{0}$, and a constant frequency offset $\Delta f_{i}$. The phase $\varphi_{i}(t)$ at time $t$ is the integration over frequency:

$$
\varphi_{i}(t)=2 \pi \int_{t_{0}}^{t} f_{i}(t) d t+\varphi_{\mathrm{ini} i}+n_{\varphi i}(t)
$$

with the initial - time independent- phase $\varphi_{\mathrm{ini}} i$, and the oscillators phase noise ${ }^{1} n_{\varphi i}(t)$ [8].

Satellite $j$ receives the signal after a delay $\tau_{i j}$ corresponding to the time it takes the signal to travel the distance $r$ between the two satellites. At the receive instance $t+\tau_{i j}$ the phase $\varphi_{j}\left(t+\tau_{i j}\right)$ of oscillator $j$ is:

$$
\varphi_{j}\left(t+\tau_{i j}\right)=2 \pi \int_{t_{0}}^{t+\tau_{i j}} f_{j}(t) d t+\varphi_{\mathrm{ini} j}+n_{\varphi j}\left(t+\tau_{i j}\right)
$$

The demodulated phase $\varphi_{j i}(t)$ available at satellite $j$ for a signal transmitted by satellite $i$ is the difference between (2) and (1) after including the system and path contributions. The data take start time $t_{0}$ can be set to zero without restricting generality. The phase differences $\varphi_{j i}(t)$ and $\varphi_{i j}(t)$ are used to obtain the compensation phase.

\section{B. Compensation Phase}

The compensation phase $\varphi_{\mathrm{c}}(t)$ is obtained by computing the difference:

$$
\varphi_{\mathrm{c}}(t)=\frac{1}{2}\left(\varphi_{21}(t)-\varphi_{12}(t)\right)
$$

where $\varphi_{21}(t)$ and $\varphi_{12}(t)$ are the demodulated phases of the synchronization signals recorded by satellite 2 and 1, respectively.

The advantage of using the phase difference - the compensation phase is actually the difference of the difference, since the phases $\varphi_{12}(t)$ and $\varphi_{21}(t)$ already represent a phase differenceis that the antenna, link path,and all common Tx/Rx system phase variations will cancel out as long as their contribution do not change within the time a pair of synchronization signals are exchanged. The compensation phase can then be used to correct the time varying oscillator phase noise errors and the frequency offset of the SAR signal.

\footnotetext{
${ }^{1}$ It is irrelevant whether $f_{i}$ is the oscillator frequency or the up-converted $\mathrm{RF}$ frequency as long as the representation of the oscillator phase noise is consistent. Consequently the term oscillator phase noise identifies the phase noise originating from the oscillator without specifying the frequency.
}

\section{Synchronization Approach and Definition of Terms}

The basic idea behind phase synchronization is to determine the phase noise and frequency offset differences in (2) and (1). Thus, the phase noise, described through its spectral density is the useful, i.e. "wanted", quantity. Here, the same analytic model and parameter values detailed in [1] are used to represent the phase noise spectral density $S_{\varphi}(f)$ at oscillator frequency $f_{\text {osc }}=10 \mathrm{MHz}$. At nominal up-converted RF frequency $f_{0}$ the valid phase noise spectral density is given by $\gamma^{2} S_{\varphi}(f)$, where $\gamma$ is the ratio of RF to oscillator frequency $\gamma=f_{0} / f_{\text {osc }}$.

A general signal flow block diagram is shown in Fig. 3 for a priori phase correction, i.e. before SAR focusing. Several factors will influence the phase of the synchronization link as shown in the lower signal path in Fig. 3. Here the transfer function $H_{\mathrm{LP}}(f)$ describes the effect of alternate pulse synchronization. The receiver noise determined by the signal-tonoise ratio $S N R$ is of special interest; its influence on the signal phase is described by the receiver phase noise spectral density function $S_{\varphi_{S N R}}(f)$. Further, for pulsed synchronization the phase is sampled, which requires a later interpolation of the compensation phase. We may choose to filter the compensation phase with an -arbitrary- transfer function $H_{\text {syn }}(f)$. Finally the compensated SAR phase (SAR phase after subtracting the compensation phase) is filtered through the azimuth compression. This filter is described through the transfer function $H_{\mathrm{az}}(f)$ and is dependent on the azimuth processing [1].

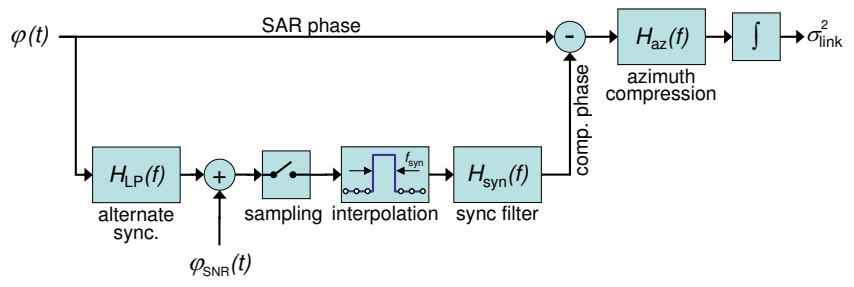

Fig. 3. Synchronization and SAR signal flow block diagram.

The performance of the synchronization link is determined by the quality of the phase noise reconstruction. The figure-ofmerit is the phase variance $\sigma_{\text {link }}^{2}$ after subtracting the oscillator phase noise and performing the azimuth compression (see Fig. 3). The link phase error, i.e. the residual contribution to the synchronized SAR, is represented by the standard deviation (STD) $\sigma_{\text {link }}$.

Note that according to the above model, the SAR transfer function $H_{\mathrm{az}}(f)$ is applied on the signal's phase instead of the signal itself. It can be shown that this is valid - from the synchronization point of view, where the SAR focusing is understood as an averaging process - for small values of the residual phase error $\sigma_{\text {link }}$.

\section{Performance of Synchronization Link}

In the following the link phase error is given for the synchronization schemes in section II including the error contributions resulting from the synchronization link itself as described in the model of section III-C. The results are given for an L-band radar with $f_{0}=1.25 \mathrm{GHz}$, i.e. with $\gamma=f_{0} / f_{\text {osc }}=125$, but the expressions are readily applicable for other frequencies. 


\section{A. Continuous Duplex Synchronization [3]}

Both satellites continuously transmit the synchronization signal during the data take. The system hardware must be capable of simultaneous transmission and reception, and the signals must be sufficiently decoupled, which may cause problems when using the same carrier frequency. Here, the single pulse length is equal to data take time $T_{\mathrm{p}}=T_{\text {data }}$, the system delay vanishes $\tau_{\mathrm{sys}}=0$, and the signal travel times are equal $\tau_{12}=\tau_{21}=\tau=r / c_{0}$ where $r$ is the distance between the two satellites. The compensation phase in (3) follows from (1) and (2) yielding:

$$
\begin{aligned}
\varphi_{\mathrm{c}}(t)= & 2 \pi\left(\Delta f_{2}-\Delta f_{1}\right) \cdot t+n_{\varphi 2}(t+\tau)-n_{\varphi 1}(t+\tau) \\
& +\pi\left(\Delta f_{2}-\Delta f_{1}\right) \cdot \tau+\varphi_{\mathrm{ini} 1}-\varphi_{\mathrm{ini} 2} \\
& +\frac{1}{2}\left(\varphi_{S N R 1}(t+\tau)-\varphi_{S N R 2}(t+\tau)\right)
\end{aligned}
$$

The first line of (4) contains the time-varying terms used to compensate the SAR phase noise and frequency offset. Here, the frequency offset term $2 \pi\left(\Delta f_{2}-\Delta f_{1}\right) t$ results in a linear phase ramp, which can be extracted to correct the frequency offset error of the SAR signal; consequently this term is neglected for the further analysis. The second line in (4) is constant for constant satellite separation and thus irrelevant for the link performance (the influence of varying satellite separation is considered in section IV-C). The main error contribution, dictating the performance of the synchronization link, is the phase variation due to receiver noise $\varphi_{S N R}$.

The receiver noise, consisting of thermal noise and the noise collected by the antenna, will introduce both amplitude and phase fluctuation to the synchronization signal. Here, the phase variations described by their spectral density function are of interest. For band limited white Gaussian noise the spectral density function $S_{\varphi_{S N R}}(f)$ is related to the $S N R$ through [9]:

$$
S_{\varphi_{S N R}}(f)=\frac{1}{2 B_{\mathrm{w}} \cdot S N R}
$$

with the receiver (noise) bandwidth $B_{\mathrm{w}}$. Choosing the value of $B_{\mathrm{w}}$ is a compromise between reducing the receiver noise (low bandwidth) on one side and ensuring that the frequency offset $\left|\Delta f_{2}-\Delta f_{1}\right|$ and the higher frequency components of $S_{\varphi}(f)$ are still within the receiver bandwidth (high bandwidth).

The compensation phase spectrum is visualized schematically in Fig. 4. In addition to the oscillator phase noise spectrum it also contains the receiver phase noise causing the link error.

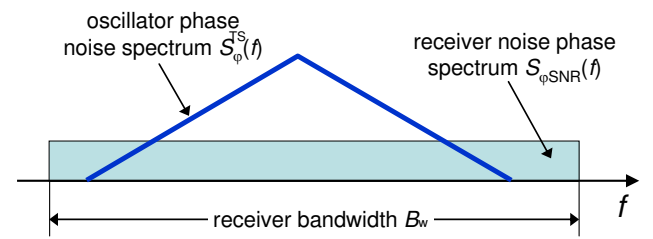

Fig. 4. Schematic spectrum of the compensation phase showing the additive white receiver noise spectrum $\left(H_{\mathrm{syn}}(f)=1\right)$.

The signal-to-noise ratio is improved through the azimuth compression where the compensated SAR signal is averaged over a period $T_{\mathrm{a}}$, which is equivalent to a low-pass filter. The link error ${ }^{2}$ variance then is:

$$
\sigma_{\text {link }}^{2}=\frac{\sigma_{S N R}^{2}}{2}=\frac{1}{4 B_{\mathrm{w}} \cdot S N R} \int_{-B_{\mathrm{w}} / 2}^{+B_{\mathrm{w}} / 2}\left|H_{\mathrm{syn}}(f) H_{\mathrm{az}}(f)\right|^{2} d f
$$

where uncorrelated noise and equal $S N R$ values are assumed for both receivers. We note that increasing the bandwidth $B_{\mathrm{w}}$ does not reduce the link error, as (6) might misleadingly suggest; the reason is that the $S N R$ is not independent from $B_{\mathrm{w}}$.

The synchronization link error $\sigma_{\text {link }}$ is plotted in Fig. 5 as a function of the $S N R$ for different azimuth integration times. Clearly, averaging reduces the error, however it should be kept in mind that $H_{\mathrm{az}}(f)$ depends on the SAR processing and is not an independent function.

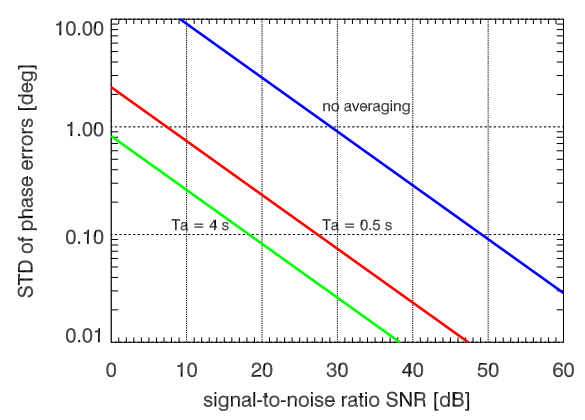

Fig. 5. Standard deviation (STD) of link error versus the $S N R$ with the averaging time $T_{\mathrm{a}}$ as parameter and a fixed bandwidth $B_{\mathrm{w}}=300 \mathrm{~Hz}$. The azimuth transfer function is $H_{\mathrm{az}}(f)=\sin \left(\pi T_{\mathrm{a}} f\right) /\left(\pi T_{\mathrm{a}} f\right)$ and $H_{\mathrm{syn}}(f)=1$.

\section{B. Pulsed Duplex Synchronization}

Synchronization pulses of duration $T_{\mathrm{p}}$ are simultaneously transmitted by both satellites every $1 / f_{\text {syn }}$ seconds. The same system hardware and decoupling constraints as for the continuous synchronization apply. Since operation is duplex, the system time delay vanishes $\tau_{\text {sys }}=0$ and the travel time is equal for both signals $\tau_{12}=\tau_{21}=\tau=r / c_{0}$. The compensation phase is the time-discrete equivalent of (4) given by:

$$
\begin{gathered}
\varphi_{\mathrm{c}}\left(t_{k}\right)=\frac{1}{2}\left(\varphi_{S N R 1}\left(t_{k}+\tau\right)-\varphi_{S N R 2}\left(t_{k}+\tau\right)\right) \\
\pi\left(\Delta f_{2}-\Delta f_{1}\right) \cdot\left(\tau+2 t_{k}\right)+n_{\varphi 2}\left(t_{k}+\tau\right)-n_{\varphi 1}\left(t_{k}+\tau\right)
\end{gathered}
$$

where the discrete sample instances $t_{k}$ are given by:

$$
t_{k}=\frac{k}{f_{\mathrm{syn}}} \quad \text { for } \quad k=0,1, \ldots\left\lfloor T_{\text {data }} \cdot f_{\mathrm{syn}}\right\rfloor
$$

with $\left\lfloor T_{\text {data }} \cdot f_{\text {syn }}\right\rfloor$ the total number of synchronization pulses during data take.

An estimate of the time continuous compensation phase $\tilde{\varphi}_{\mathrm{c}}(t)$ will be recovered from the discrete samples (for example by sinc interpolation). Now, in addition to the receiver noise, the compensation phase will contain interpolation and aliasing errors (also detailed in [1]) as shown schematically in Fig. 6.

\footnotetext{
${ }^{2}$ The filter mismatch error (introduced later in section IV-C) is ignored at this point, thus assuming no additional distortion within the link path $\left(H_{\mathrm{syn}}(f)=\right.$ 1 ); the filter mismatch error is obtained readily by (17) by setting $H_{\mathrm{LP}}(f)=1$ and replacing the upper integral limit by $B_{\mathrm{w}} / 2$.
} 


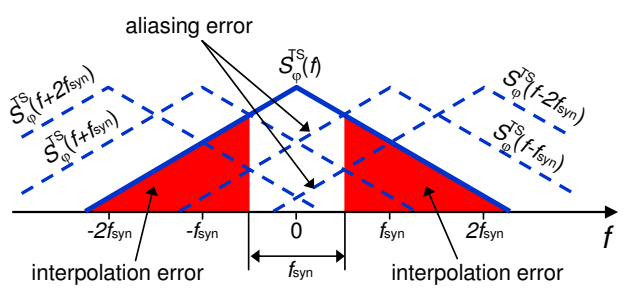

Fig. 6. Schematic spectrum of the compensation phase showing the aliasing and interpolating errors. The receiver noise, although present, is not shown.

The link variance is the sum of the interpolating, aliasing, and receiver noise variances, with the interpolation variance

$$
\sigma_{\mathrm{i}}^{2}=2 \gamma^{2} \int_{f_{\mathrm{syn}} / 2}^{\infty} S_{\varphi}(f)\left|H_{\mathrm{az}}(f)\right|^{2} d f,
$$

the aliasing variance

$$
\sigma_{\mathrm{a}}^{2}=2 \gamma^{2} \sum_{i=1}^{\infty} \int_{-f_{\mathrm{syn}} / 2}^{f_{\mathrm{syn}} / 2} S_{\varphi}\left(f+i \cdot f_{\mathrm{syn}}\right)\left|H_{\mathrm{syn}}(f) H_{\mathrm{az}}(f)\right|^{2} d f
$$

and the receiver noise variance

$$
\frac{1}{2} \sigma_{S N R}^{2}=\frac{1}{4 f_{\mathrm{syn}} \cdot S N R} \int_{-f_{\mathrm{syn} / 2}}^{f_{\mathrm{syn}} / 2}\left|H_{\mathrm{syn}}(f) H_{\mathrm{az}}(f)\right|^{2} d f
$$

The interpolation error is because frequency components outside the range $-\frac{1}{2} f_{\text {syn }}<f<+\frac{1}{2} f_{\text {syn }}$ are lost due to the sampling and hence can not be reconstructed. The aliasing error results from the cyclic repetition of the spectrum at integer multiples of the sampling frequency $f_{\text {syn }}$; thus, frequency components outside the range $-\frac{1}{2} f_{\text {syn }}<f<+\frac{1}{2} f_{\text {syn }}$ will be folded into the original spectrum causing the aliasing error.

The individual contributions to the total link error are shown in Fig. 7. The link error is dictated by the interpolation and aliasing errors for low $f_{\text {syn }}$ and by the receiver noise for high $f_{\text {syn }}$. Without averaging, Fig. 7(a), the interpolation and aliasing errors are equal $\sigma_{\mathrm{i}}=\sigma_{\mathrm{a}}$. Averaging mainly reduces the receiver noise and the interpolation error as shown in Fig. 7(b), and thus results in a smaller link error.

\section{Pulsed Alternate Synchronization}

The transmit instance of satellite 2 is delayed by $\tau_{\text {sys }}$ with respect to satellite 1 . In this case a full-duplex system hardware is dispensable, and the signals are inherently decoupled offering the possibility of using a single carrier frequency. The pulsed alternate scheme has been suggested within the context of navigation satellite state determination in [10]. Each satellite transmits/receives pulses of duration $T_{\mathrm{p}}$ at the synchronization rate $f_{\text {syn }}$. The time discrete compensation phase is

$$
\begin{aligned}
\varphi_{\mathrm{c}}\left(t_{k}\right)= & \frac{1}{2}\left(\varphi_{S N R 1}\left(t_{k}+\tau+\tau_{\mathrm{sys}}\right)-\varphi_{S N R 2}\left(t_{k}+\tau\right)\right) \\
& \pi\left(\Delta f_{2}-\Delta f_{1}\right) \cdot\left(\tau+\tau_{\mathrm{sys}}+2 t_{k}\right)-\pi f_{D} \tau_{\mathrm{sys}} \\
& +n_{\varphi 2}\left(t_{k}+\tau\right)+n_{\varphi 2}\left(t_{k}+\tau_{\mathrm{sys}}\right) \\
& -n_{\varphi 1}\left(t_{k}+\tau+\tau_{\mathrm{sys}}\right)-n_{\varphi 1}\left(t_{k}\right)
\end{aligned}
$$

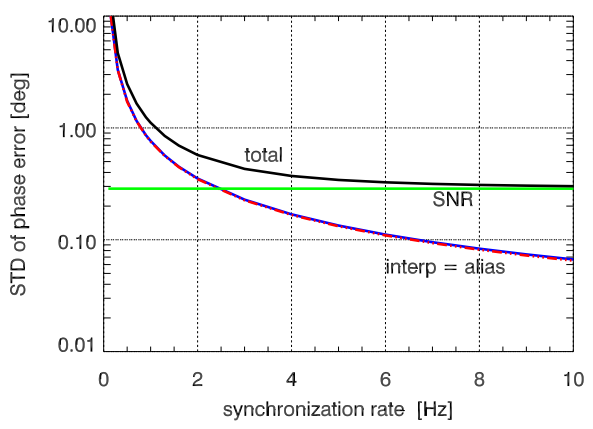

(a) $T_{\mathrm{a}}=0 \mathrm{~s}$

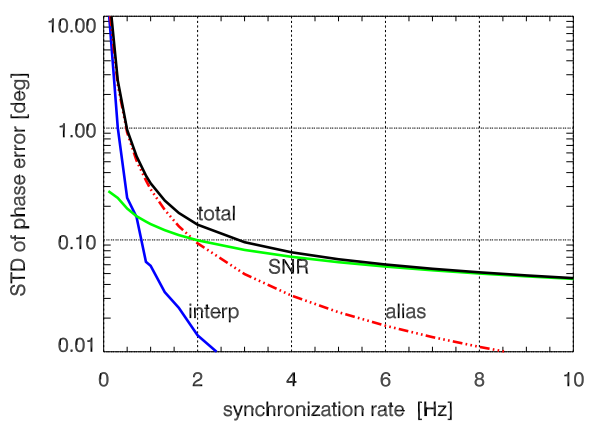

(b) $T_{\mathrm{a}}=4 \mathrm{~s}$

Fig. 7. Contributions to the link error (standard deviation) versus the synchronization rate $f_{\text {syn }}$ for $S N R=40 \mathrm{~dB}$.

where $f_{D}=f_{0} v_{\text {sat }} / c_{0}$ is the Doppler frequency due to the relative velocity $v_{\text {sat }}$ between the two satellites. The Doppler phenomenon manifests itself for alternate synchronization pulses, because of the unequal signal travel times $\tau_{12} \neq \tau_{21}$ due to the changing satellite separation between the transmit instances $t$ and $t+\tau_{\text {sys }}$. However, the Doppler phase contribution is constant for constant $v_{\text {sat }}$. Only a relative satellite acceleration, i.e. a time dependent $v_{\text {sat }}(t)$ will cause a phase error. For severe intersatellite acceleration a Doppler phase compensation is necessary which requires the satellite separation to be known. The last two lines in (12) represent the wanted compensation phase terms, however, it is recognized that each oscillator's phase noise is sampled at different time instances. This is equivalent to a lowpass comb filter having the following impulse response and transfer function, respectively ${ }^{3}$ :

$$
\begin{aligned}
& h_{\mathrm{LP}}\left(t_{k}\right)=\frac{1}{2}\left(\delta\left(t_{k}\right)+\delta\left(t_{k}+\tau_{\mathrm{sys}}\right)\right) \\
& H_{\mathrm{LP}}(f)=\exp \left(-j \pi f \tau_{\mathrm{sys}}\right) \cdot \cos \left(\pi f \tau_{\mathrm{sys}}\right)
\end{aligned}
$$

Thus the compensation phase will contain a low-pass filtered version of the oscillator noise. On a first sight this will cause an increase of the phase error. However, as indicated in Fig. 8 the low-pass filter effect may prove useful for reducing the aliasing error, since it attenuates the higher frequency components $|f|>\frac{1}{2} f_{\text {syn }}$ of the oscillator phase noise, which would otherwise contribute to the aliasing error.

The total phase variance is the sum given by:

$$
\sigma_{\text {link }}^{2}=\sigma_{\mathrm{i}}^{2}+\sigma_{\mathrm{a}}^{2}+\sigma_{\mathrm{f}}^{2}+\frac{1}{2} \sigma_{S N R}^{2}
$$

\footnotetext{
${ }^{3}$ Here the time delay $\tau$ is ignored because it is assumed much shorter in comparison to the system delay, i.e. $\tau \ll \tau_{\text {sys }}$.
} 


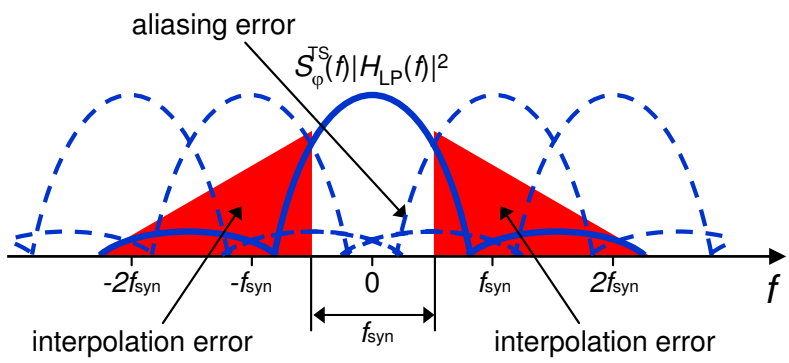

Fig. 8. Schematic spectrum of the compensation phase showing the low-pass filtered spectrum and the aliasing and interpolating errors.

with interpolation and receiver noise variance given by (9) and (11), respectively. The aliasing variance is given by:

$$
\begin{aligned}
\sigma_{\mathrm{a}}^{2}= & 2 \gamma^{2} \sum_{i=1}^{\infty} \int_{-f_{\mathrm{syn}} / 2}^{f_{\mathrm{syn}} / 2} S_{\varphi}\left(f+i \cdot f_{\mathrm{syn}}\right) \cdot \\
& \left|H_{\mathrm{LP}}\left(f+i \cdot f_{\mathrm{syn}}\right) H_{\mathrm{syn}}(f) H_{\mathrm{az}}(f)\right|^{2} d f
\end{aligned}
$$

In additional a filter mismatch error $\sigma_{\mathrm{f}}$ caused by the distortions of the synchronization link transfer function $H_{\text {syn }}(f)$ may appear. This is given by:

$$
\sigma_{\mathrm{f}}^{2}=2 \gamma^{2} \int_{0}^{f_{\mathrm{syn}} / 2} S_{\varphi}(f)\left|H_{\mathrm{az}}(f)\right|^{2} \cdot\left|H_{\mathrm{LP}}(f) H_{\mathrm{syn}}(f)-1\right|^{2} d f
$$

The link error for the case of pulsed alternate synchronization is shown in Fig. 9. The synchronization system transfer function is chosen to eliminate the filter mismatch error, hence $H_{\mathrm{LP}}(f) H_{\mathrm{syn}}(f)=1$ for $|f| \leq \frac{1}{2} f_{\mathrm{syn}}$. The main difference between alternate and duplex synchronization is the aliasing error (compare Fig. 7(b) to Fig. 9). This error is reduced for $f_{\text {syn }}$ values near $1 / 2 \tau_{\text {sys }}$. Thus for a fixed system delay $\tau_{\text {sys }}=25 \mathrm{~ms}$ in Fig. 9(a), the aliasing error is only reduced for rather high $f_{\text {syn }} \approx 20 \mathrm{~Hz}$. In this case the total error, mainly dictated by the receiver noise is not significantly reduced. When adapting $\tau_{\text {sys }}$ to the synchronization rate so as to maximize the low-pass filter effect $\tau_{\text {sys }}=1 / 2 f_{\text {syn }}$ (in this case the transmit instances of satellite 1 are interleaved midway between those of satellite 1) the aliasing error is minimized ${ }^{4}$ and the total link error can be reduced for small $f_{\text {syn }}$ values as seen from Fig. 9(b).

\section{CONCLUSION}

A synchronization link is a solution to avert the performance degradation due to oscillator phase noise in bistatic SAR. Starting with an investigation of different synchronization schemes closed analytic expression for the link performance are derived. The approach is novel in the sense that it utilizes the knowledge of the statistical oscillator parameters, system error contributions, sampling considerations and SAR processing parameters in order to formulate the residual phase error after synchronization. The results showed, that a careful consideration of the interrelation between the various synchronization link parameters is required. Successful oscillator phase

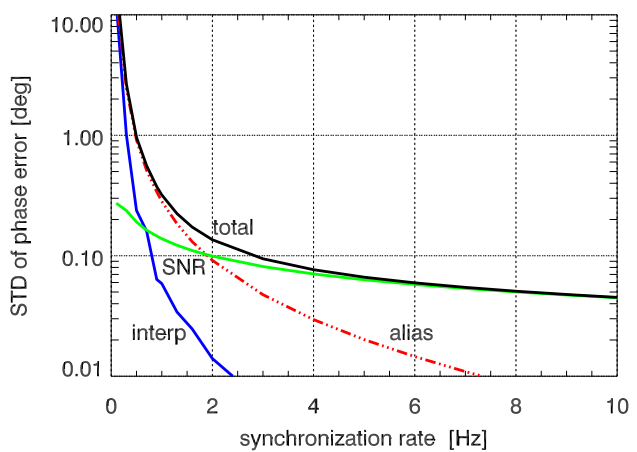

(a) fixed $\tau_{\text {sys }}=25 \mathrm{~ms}$

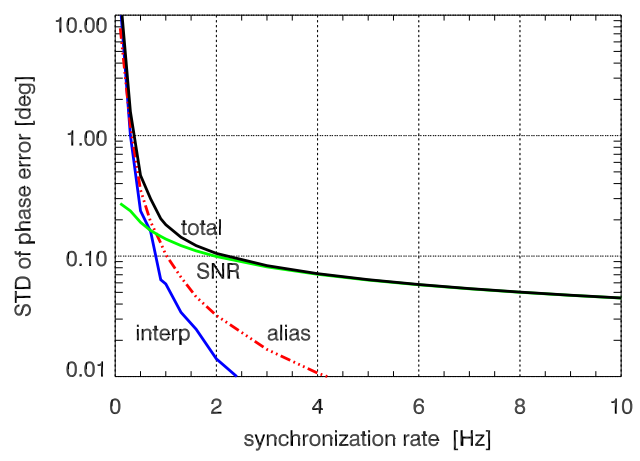

(b) optimized $\tau_{\text {sys }}=1 / 2 f_{\text {syn }}$

Fig. 9. Contributions to the link error versus the synchronization rate $f_{\text {syn }}$ for $S N R=40 \mathrm{~dB}, T_{\mathrm{a}}=4 \mathrm{~s}$ and $H_{\mathrm{LP}}(f) H_{\mathrm{syn}}(f)=1$.

noise compensation is possible, if the compensation algorithm and signal timing is adapted to the link hardware and SAR parameters.

\section{REFERENCES}

[1] G. Krieger and M. Younis, "Impact of oscillator noise in bistatic and multistatic SAR,' IEEE Geoscience and Remote Sensing Letters, submitted for publication, 2005.

[2] G. Krieger, M. R. Cassola, M. Younis, and R. Metzig, "Impact of oscillator noise in bistatic and multistatic SAR," in Proc. Int. Geoscience and Remote Sensing Symposium IGARSS'2005, Seoul, Korea, July 2005.

[3] M. Eineder, "Ocillator clock drift compensation in bistatic interferometric SAR," in Proc. Int. Geoscience and Remote Sensing Symposium IGARSS'2003, Tolouse, France, July 2003.

[4] M. Weiß, "Time and frequency synchronisation aspects for bistatic SAR systems," in Proc. European Conference on Synthetic Aperture Radar EUSAR'2004, May 2004.

[5] J. Kim and B. D. Tapley, "Simulation of dual one-way ranging measurements," Journal of Spacecraft and Rockets, vol. 40, no. 3, May 2003.

[6] J. L. MacArthur and A. S. Posner, "Satellite-to-satellite range-rate measurements," IEEE Transactions on Geoscience and Remote Sensing, vol. 23, no. 4, pp. 517-523, July 1985.

[7] A. Papoulis, "Error analysis in sampling theory," Proceedings of the IEEE, vol. 54, no. 7, pp. 947-955, July 1966.

[8] J. Rutman, "Characterization of phase and frequency instabilities in precision frequency sources: Fifteen years of progress," Proceedings of the IEEE, vol. 66, no. 9, pp. 1048-1074, Sept. 1978.

[9] F. G. Stremler, Introduction to Communication Systems, 2nd ed. AddisonWesley, 1982.

[10] B. Eissfeller, T. Zink, R. Wolf, J. Hammesfahr, A. Hornbostel, J. Hahn, and P. Tavella, "Autonomous satellite state determination by use of two-directional links," International Journal of Satelite Communications, vol. 18 , no. 4,2000 .

\footnotetext{
${ }^{4}$ True for a strictly monotonic decreasing phase noise spectrum $S_{\varphi}(f)$.
} 